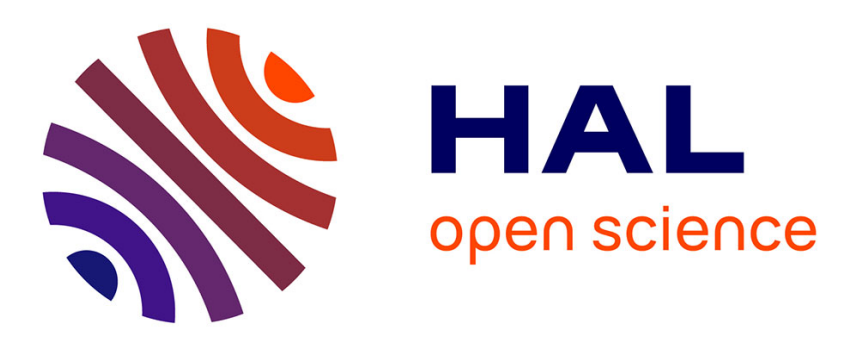

\title{
A Framework for Wind Sensitivity Analysis for Trajectory Tracking
}

\author{
Hector Escamilla Nuñez, Hakim Bouadi, Felix Mora-Camino
}

\section{To cite this version:}

Hector Escamilla Nuñez, Hakim Bouadi, Felix Mora-Camino. A Framework for Wind Sensitivity Analysis for Trajectory Tracking. AIAA GNC 2017, AIAA Guidance, Navigation, and Control Conference, AIAA, Jan 2017, Grapevine, United States. 10.2514/6.2017-1726 . hal-01429728

\section{HAL Id: hal-01429728 \\ https://hal-enac.archives-ouvertes.fr/hal-01429728}

Submitted on 24 Mar 2017

HAL is a multi-disciplinary open access archive for the deposit and dissemination of scientific research documents, whether they are published or not. The documents may come from teaching and research institutions in France or abroad, or from public or private research centers.
L'archive ouverte pluridisciplinaire HAL, est destinée au dépôt et à la diffusion de documents scientifiques de niveau recherche, publiés ou non, émanant des établissements d'enseignement et de recherche français ou étrangers, des laboratoires publics ou privés. 


\title{
A Framework for Wind Sensitivity Analysis for Trajectory Tracking.
}

\author{
H. Escamilla Núñez \\ hector.hen91@gmail.com \\ H. Bouadi \\ hakimbouadi@yahoo.fr \\ F. Mora Camino \\ felix.mora@enac.fr
}

\begin{abstract}
In this paper a sensitivity analysis of trajectories w.r.t. wind effects is proposed. This analysis will allow to specify wind estimates properties as well as control reactiveness characteristics to ensure accurate guidance in real atmosphere. The mathematic model developed for flight dynamics is intended to take into account wind gust effects on AoA, sideslip angle and airspeed. A two layer Nonlinear Dynamic Inversion (NDI) structure has been considered to control fast and slow dynamics. In order to test the proposed approach, a full six degrees of freedom Matlab model with trained Neural Networks to emulate a Boeing 737-200 was developed.
\end{abstract}

\section{NOMENCLATURE}

$V_{a}$

$\eta=[\phi, \theta, \psi]^{T}$

$\Omega=[p, q, r]^{T}$

$u, v, w$

$F_{t h r}, L, D, Y$

$F_{x_{a}}, F_{y_{a}}, F_{z_{a}}$

$L^{\prime}, M, N$

$C_{L}, C_{D}, C_{Y}$

$C_{l}, C_{m}, C_{n}$

$\mu$

$g$

$m$

$\rho$

$S$

$b$

$\bar{c}$

A

Angle of Attack, deg

Sideslip angle, deg

Airspeed, $\mathrm{m} / \mathrm{s}$

Euler angles, deg

Angular velocities, $\mathrm{deg} / \mathrm{s}$

Groundspeed in the body frame, $\mathrm{m} / \mathrm{s}$

Thrust/Lift/Drag/Sideforce, $N$

Aerodynamic Forces in the body frame, $N$

Rolling/Pitching/Yawing moments, $N \cdot m$

Lift/Drag/Sideforce aerodynamic coefficients

Rolling/Pitching/Yawing aerodynamic coefficients

Thrust specific fuel consumption, $\mathrm{kg} /(N \cdot s)$

Gravity, $m / s^{2}$

Aircraft mass, $k g$

Air density, $\mathrm{kg} / \mathrm{m}^{3}$

Wing area, $123.55 \mathrm{~m}^{2}$

Wingspan, $28.34 \mathrm{~m}$

Mean chord, $4.35 \mathrm{~m}$

Inertia matrix component, 1, 278, 369.56 $\mathrm{kg} \cdot \mathrm{m}^{2}$

Ph.D student at MAIAA, ENAC, 7 avenue Edouard Belin, Toulouse, 31055, France.

Ecole Militaire Polytechnique, Bordj-El-Bahri, 16111, Alger, Algeria.

Head of Automation Research Group at MAIAA, ENAC, 7 avenue Edouard Belin, Toulouse, 31055, France. 


$\begin{array}{ll}B & \text { Inertia matrix component, } 3,781,267.79 \mathrm{~kg} \cdot \mathrm{m}^{2} \\ C & \text { Inertia matrix component, } 4,877,649.98 \mathrm{~kg} \cdot \mathrm{m}^{2} \\ E & \text { Inertia matrix component, } 135,588.17 \mathrm{~kg} \cdot \mathrm{m}^{2} \\ h_{\max } & \text { Service ceiling, } 10,700 \mathrm{~m} \\ V_{\max } & \text { Maximum speed, } 0.85 \mathrm{Mach} \\ X_{c g} & \text { Aircraft cg position, } 15.3 \mathrm{~m} \\ Y_{c g} & \text { Aircraft cg position, } 0 \mathrm{~m} \\ Z_{c g} & \text { Aircraft cg position, }-1.016 \mathrm{~m}\end{array}$

\section{INTRODUCTION}

As air traffic is predicted to increase dramatically in the upcoming years, new problems and requirements are arising, among those involving the use of 4D trajectories are of most interest. NextGen[1] and SESAR[2] control projects, adopt the Trajectory Based Operations (TBO) paradigm, where safety and traffic capacity issues are the milestones. Knowing that TBO relies on 4D guidance, it is not surprising that automation becomes one of the important enablers, allowing aircraft to follow more accurately a desired trajectory, resulting in a fuel usage decrease and reduced $\mathrm{CO}_{2}$ emissions per flight. It is expected that 4D guidance improves safety by decreasing the occurrence of near mid-air collisions for planned conflict free 4D trajectories and in consequence, decrease the work load for air traffic controllers .

Considering that one of the main disturbances during flight are wind gusts, it seems natural to take into account their effects while modelling aircraft dynamics. Once a mathematic model with wind contributions is obtained, a flight control structure must be assumed. In this work, a two-level Nonlinear Dynamic Inversion (NDI) has been developed to control the position and the angular velocities. The approach consists in transform algebraically a nonlinear system into a system with linear dynamics [3], [4]. Separation of slow and fast dynamics are common practice [5], [6]. Also, some machine learning algorithms and/or variations of the classical NDI approach have been tested in order to improve the performance of the method and provide robustness to uncertainties like modeling and/or measuring errors, showing good simulation results [7], [8]. However, full knowledge of variables and parameters cannot be acquired due to possible corrupted measures or uncertainty in parameters, leading to guidance inaccuracy. Thus, this work will provide a tool to quantify the performance of the aircraft in terms of position errors due to wind gusts by performing a sensitivity analysis through simulation. Actuator dynamics for the control surfaces involving a time-constant will also be considered.

Methods to perform sensitivity analysis such as screening are common [9], where a baseline experiment is done using nominal values of parameters, then, selecting two extreme values of these, the results of the perturbed experiment w.r.t. the baseline are observed in order to decide to which parameters the model is most sensitive to. Nevertheless, interaction between parameters is neglected and the correlation between them and outputs of the system is considered linear. Variance-based approaches show how to find which are the most relevant parameters and how much they affect the system output by computing sensitivity indices such as Sobol indices, quantifying the amount of variance in the output caused by a parameter. These methods allow interaction of different parameters and nonlinear responses between them and the outputs [10]. Another technique is to compute the partial derivatives of the interesting variables to analyze the local sensitivity [11], [12]. For our work, using an approach similar to screening analysis, wind information is used as a changing parameter to quantify the effects it has on the aircraft position.

The paper is organized as follows, Section II provides the developed mathematical model under wind conditions. Section III describes the adopted NDI control structure and Section IV shows the simulation 
results of this control law. Section V shows the wind sensitivity analysis trough simulation. Finally, conclusions are described in Section VI.

\section{Dynamic Model in Wind Conditions}

Wind gusts contributions are considered to affect directly the aircraft angle of attack (AoA), sideslip angle and airspeed, and then their effects are propagated to the angular velocities, then to the attitude, and finally to the position. Hence, when wind effects are considered to be reflected only in attitude, or only in position, it may differ from the actual phenomena.

\section{A. Adopted Frames and Flight Variables}

The earth reference frame is assumed to be Earth centered and denoted by $F_{E}=\left(O_{E}, x_{E}, y_{E}, z_{E}\right)$. A second reference frame is considered to represent the fast dynamics of the aircraft, it is attached to the body's c.g. and is defined as $F_{B}=\left(C, x_{B}, y_{B}, z_{B}\right)$, referred to it as the body frame. The $x_{B}$ axis goes from tail to nose of the aircraft while the $z_{B}$ axis, perpendicular to the latter, points downwards and lies in the symmetry plane of the aircraft, to complete the triad, the direction of $y_{B}$ can be obtained by the cross product $\overrightarrow{z_{B}} \times \overrightarrow{x_{B}}$. Lastly, denoted by $F_{W}=\left(C, x_{W}, y_{W}, z_{W}\right)$, a wind frame is defined in order to represent the aerodynamic actions on the aircraft. This frame has its $x_{W}$ axis aligned with the aircraft velocity vector relative to the surrounding air mass, i.e. the airspeed. The difference between $x_{B}$ and the projection of $x_{W}$ in the $C x_{B} z_{B}$ plane, gives birth to the angle of attack. Also, the angle created by the projection of $V_{a}$ in the $C x_{B} y_{B}$ plane and the $x_{B}$ axis, is known as the sideslip angle. Physical quantities from the wind frame can be mapped into the body frame by the following rotation matrix:

$$
L_{B W}=\left[\begin{array}{ccc}
c_{\alpha} c_{\beta} & -c_{\alpha} s_{\beta} & -s_{\alpha} \\
s_{\beta} & c_{\beta} & 0 \\
s_{\alpha} c_{\beta} & -s_{\alpha} s_{\beta} & c_{\alpha}
\end{array}\right]
$$

In the same tenor, the velocity of the aircraft $\left(V_{E}=\left[\dot{x_{E}}, \dot{y_{E}}, \dot{z_{E}}\right]^{T}\right)$, is expressed in the body frame as $V_{B}=[u, v, w]^{T}$. Also, the euler angles are used to describe the attitude of the aircraft. These angles are bounded as follows $\phi\{-\pi, \pi\} ; \theta\left\{-\frac{\pi}{2}, \frac{\pi}{2}\right\} ; \psi\{-\pi, \pi\}$, although limits are never reached during normal operation. The rotation matrix from the body to the earth frame considering a rotation around the axes in the order $z y x$, is given by:

$$
L_{E B}=\left[\begin{array}{ccc}
c_{\theta} c_{\psi} & s_{\phi} s_{\theta} c_{\psi}-c_{\phi} s_{\psi} & c_{\phi} s_{\theta} c_{\psi}+s_{\phi} s_{\psi} \\
c_{\theta} s_{\psi} & s_{\phi} s_{\theta} s_{\psi}+c_{\phi} c_{\psi} & c_{\phi} s_{\theta} s_{\psi}-s_{\phi} c_{\psi} \\
-s_{\theta} & s_{\phi} c_{\theta} & c_{\phi} c_{\theta}
\end{array}\right]
$$

Moreover, defining the wind speed in the body frame as $V_{w}=\left[V_{w_{x}}, V_{w_{x}}, V_{w_{x}}\right]^{T}$ and from the rigid-body equations and other known relations extracted from [13], [14] it is obtained:

$$
\begin{gathered}
{\left[\begin{array}{c}
u \\
v \\
w
\end{array}\right]=\left[\begin{array}{c}
V_{a} c_{\alpha} c_{\beta}+V_{w_{x}} \\
V_{a} s_{\beta}+V_{w_{y}} \\
V_{a} s_{\alpha} c_{\beta}+V_{w_{z}}
\end{array}\right]} \\
{\left[\begin{array}{c}
\dot{u} \\
\dot{v} \\
\dot{w}
\end{array}\right]=\left[\begin{array}{c}
\frac{1}{m}\left(F_{x_{a}}+F_{t h r}\right)-g s_{\theta}+r v-q w \\
\frac{1}{m} F_{y_{a}}+g c_{\theta} s_{\phi}+p w-r u \\
\frac{1}{m} F_{z_{a}}+g c_{\theta} c_{\phi}+q u-p v
\end{array}\right]} \\
\alpha=\arctan \left(\frac{w}{u}\right) \\
\beta=\arcsin \left(\frac{v}{V_{a}}\right) \\
V_{a}=\sqrt{u^{2}+v^{2}+w^{2}}
\end{gathered}
$$

The vector $R=\left[\alpha, \beta, V_{a}\right]^{T}$ is defined for simplicity in further equations. 


\section{B. Attitude Dynamics}

Concerning the attitude of the aircraft, the angular rates are produced by the deflection of ailerons, elevator and rudder, denoted by $\left[\delta_{a i l}, \delta_{e l e}, \delta_{r u d}\right]^{T}$ respectively. Thus, the rotational equations of the aircraft are given by:

$$
\dot{\Omega}=I^{-1} M_{e x t}-I^{-1} \Omega \times(I \Omega)
$$

where $M_{\text {ext }}$ denotes the rolling, pitching and yawing moments, and $I$ stands for the inertia matrix, considered constant by neglecting the weight change of the aircraft:

$$
I=\left[\begin{array}{ccc}
A & 0 & -E \\
0 & B & 0 \\
-E & 0 & C
\end{array}\right]
$$

Thereby, introducing relations for the aerodynamic moments:

$$
\left[\begin{array}{c}
L^{\prime} \\
M \\
N
\end{array}\right]=\frac{1}{2} \rho S V_{a}^{2}\left(\left[\begin{array}{c}
b C_{l} \\
\bar{c} C_{m} \\
b C_{n}
\end{array}\right]+C_{\delta}\left[\begin{array}{c}
\delta_{\text {ail }} \\
\delta_{\text {ele }} \\
\delta_{\text {rud }}
\end{array}\right]\right)
$$

where

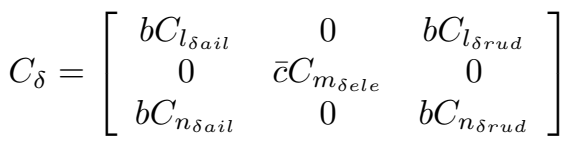

The rolling, pitching and yawing aerodynamic coefficients are denoted by:

$$
\left[\begin{array}{c}
C_{l} \\
C_{m} \\
C_{n}
\end{array}\right]=\left[\begin{array}{c}
C_{l_{\beta}} \beta+C_{l_{p}} \frac{b p}{2 V_{a}}+C_{l_{r}} \frac{b r}{2 V_{a}} \\
C_{m_{0}}+C_{m_{\alpha}} \alpha+C_{m_{q}} \frac{c q}{2 V_{a}} \\
C_{n_{\beta}} \beta+C_{n_{p}} \frac{b p}{2 V_{a}}+C_{n_{r}} \frac{b r}{2 V_{a}}
\end{array}\right]
$$

Then, equation (6) can be rewritten as in [15] like.

$$
\left[\begin{array}{c}
\dot{p} \\
\dot{q} \\
\dot{r}
\end{array}\right]=\frac{1}{2} \rho V_{a}^{2} S I^{-1}\left(\left[\begin{array}{c}
b C_{l} \\
\bar{c} C_{m} \\
b C_{n}
\end{array}\right]+C_{\delta}\left[\begin{array}{c}
\delta_{\text {ail }} \\
\delta_{\text {ele }} \\
\delta_{\text {rud }}
\end{array}\right]\right)-I^{-1}\left[\begin{array}{c}
p \\
q \\
r
\end{array}\right] \times\left(I\left[\begin{array}{c}
p \\
q \\
r
\end{array}\right]\right)
$$

Subsequently, differentiating (5a), the rate of change of the angle of attack is given by:

$$
\begin{aligned}
\dot{\alpha}= & \frac{u \dot{w}-w \dot{u}}{u^{2}+w^{2}} \\
= & \frac{\left(V_{a} c_{\beta}\right)^{2}\left[q-\tan _{\beta}\left(p c_{\alpha}+r s_{\alpha}\right)\right]+V_{a} c_{\beta}\left[g_{1}-\frac{1}{m}\left(L+F_{t h r} s_{\alpha}\right)\right]+V_{w_{x}}\left(\frac{F_{z_{a}}}{m}+g c_{\theta} c_{\phi}\right)-V_{w_{z}}\left(\frac{F_{x_{a}}}{m}+\frac{F_{t h r}}{m}-g s_{\theta}\right)}{\left(V_{a} c_{\beta}\right)^{2}+2 V_{a} c_{\beta}\left(V_{w_{x}} c_{\alpha}+V_{w_{z}} s_{\alpha}\right)+V_{w_{x}}^{2}+V_{w_{z}}^{2}} \\
& \frac{-V_{w_{x}} V_{w_{y}} p-V_{w_{y}} V_{w_{z}} r+q\left(V_{w_{x}}^{2}+V_{w_{z}}^{2}\right)+V_{a} c_{\beta}\left[V_{w_{x}}\left(2 q c_{\alpha}-p \tan _{\beta}\right)-V_{w_{y}}\left(p c_{\alpha}+r s_{\alpha}\right)+V_{w_{z}}\left(2 q s_{\alpha}-r \tan _{\beta}\right)\right]}{}
\end{aligned}
$$

with:

$$
g_{1}=g\left(c_{\alpha} c_{\theta} c_{\phi}+s_{\alpha} s_{\theta}\right)
$$

Similarly, differentiating (5b), the rate of change of the sideslip angle is given by:

$$
\begin{aligned}
\dot{\beta}= & \frac{V_{a} \dot{v}-v \dot{V}_{a}}{V_{a} \sqrt{V_{a}^{2}-v^{2}}} \\
= & \frac{V_{a}^{2} c_{\beta}\left[\frac{1}{m}\left(Y-F_{t h r} c_{\alpha} s_{\beta}\right)+g_{2}+V_{a}\left(p s_{\alpha}-r c_{\alpha}\right)\right]+V_{a}^{2}\left(V_{w_{z}} p-V_{w_{x}} r\right)+\left(\frac{F_{y_{a}}}{m}+g c_{\theta} s_{\phi}\right)\left[2 V_{a} c_{\beta}\left(V_{w_{x}} c_{\alpha}+V_{w_{z}} s_{\alpha}\right)\right.}{\left\{V_{a}^{2}+2 V_{a}\left[V_{w_{y}} s_{\beta}+c_{\beta}\left(V_{w_{x}} c_{\alpha}+V_{w_{z}} s_{\alpha}\right)\right]+V_{w_{x}}^{2}+V_{w_{y}}^{2}+V_{w_{z}}^{2}\right\} \sqrt{V_{a}^{2} c_{\beta}^{2}-V_{w_{y}}\left(2 V_{a} s_{\beta}+V_{w_{y}}\right)}} \\
& \frac{\left.+V_{w_{x}}^{2}+V_{w_{z}}^{2}\right]+\left[V_{a} c_{\beta}\left(p s_{\alpha}-r c_{\alpha}\right)+V_{w_{z}} p-V_{w_{x}} r\right]\left\{2 V_{a}\left[V_{w_{y}} s_{\beta}+c_{\beta}\left(V_{w_{x}} c_{\alpha}+V_{w_{z}} s_{\alpha}\right)\right]+V_{w_{x}}^{2}+V_{w_{y}}^{2}+V_{w_{z}}^{2}\right\}}{} \\
& -\left(\frac{F_{z_{a}}}{m}+g c_{\theta} c_{\phi}\right)\left[V_{a}\left(V_{w_{y}} s_{\alpha} c_{\beta}+V_{w_{z}} s_{\beta}\right)+V_{w_{y}} V_{w_{z}}\right]-\left(\frac{F_{x_{a}}}{m}+\frac{F_{t h r}}{m}-g s_{\theta}\right)\left[V_{a}\left(V_{w_{y}} c_{\alpha} c_{\beta}+V_{w_{x}} s_{\beta}\right)+V_{w_{x}} V_{w_{y}}\right]
\end{aligned}
$$


with:

$$
g_{2}=g\left(c_{\beta} c_{\theta} s_{\phi}+s_{\beta} c_{\alpha} s_{\theta}-s_{\alpha} s_{\beta} c_{\theta} c_{\phi}\right)
$$

Then, from $(5 \mathrm{c})$, it is obtained:

$$
\begin{aligned}
\dot{V}_{a} & =\frac{u \dot{u}+v \dot{v}+w \dot{w}}{V_{a}} \\
& =g_{3}+\frac{1}{m}\left(F_{t h r} c_{\alpha} c_{\beta}-D\right)+\frac{1}{m V_{a}}\left[V_{w_{x}}\left(F_{x_{a}}+F_{t h r}-m g s_{\theta}\right)+V_{w_{y}}\left(F_{y_{a}}+m g c_{\theta} s_{\phi}\right)+V_{w_{z}}\left(F_{z_{a}}+m g c_{\theta} c_{\phi}\right)\right]
\end{aligned}
$$

with:

$$
g_{3}=g\left(-c_{\alpha} c_{\beta} s_{\theta}+s_{\beta} c_{\theta} s_{\phi}+s_{\alpha} c_{\beta} c_{\theta} c_{\phi}\right)
$$

The expression in matrix form of relations (11), (12) and (13), rearranged for $\Omega$ is denoted by:

$$
\left[\begin{array}{c}
\dot{\alpha} \\
\dot{\beta} \\
\dot{V}_{a}
\end{array}\right]=\left[\begin{array}{ccc}
H_{11} & 1 & H_{13} \\
H_{21} & 0 & H_{23} \\
0 & 0 & 0
\end{array}\right]\left[\begin{array}{c}
p \\
q \\
r
\end{array}\right]+\left[\begin{array}{c}
Q_{1} \\
Q_{2} \\
Q_{3}
\end{array}\right]
$$

where

$$
\begin{gathered}
H_{11}=\frac{-\left(V_{a} c_{\alpha} c_{\beta}+V_{w_{x}}\right)\left(V_{a} s_{\beta}+V_{w_{y}}\right)}{\left(V_{a} c_{\beta}\right)^{2}+2 V_{a} c_{\beta}\left(V_{w_{x}} c_{\alpha}+V_{w_{z}} s_{\alpha}\right)+V_{w_{x}}^{2}+V_{w_{z}}^{2}} \\
H_{13}=\frac{-\left(V_{a} s_{\beta}+V_{w_{y}}\right)\left(V_{a} s_{\alpha} c_{\beta}+V_{w_{z}}\right)}{\left(V_{a} c_{\beta}\right)^{2}+2 V_{a} c_{\beta}\left(V_{w_{x}} c_{\alpha}+V_{w_{z}} s_{\alpha}\right)+V_{w_{x}}^{2}+V_{w_{z}}^{2}} \\
H_{21}=\frac{V_{a} s_{\alpha} c_{\beta}+V_{w_{z}}}{\sqrt{V_{a}^{2} c_{\beta}^{2}-V_{w_{y}}\left(2 V_{a} s_{\beta}+V_{w_{y}}\right)}} \\
H_{23}=\frac{-\left(V_{a} c_{\alpha} c_{\beta}+V_{w_{x}}\right)}{\sqrt{V_{a}^{2} c_{\beta}^{2}-V_{w_{y}}\left(2 V_{a} s_{\beta}+V_{w_{y}}\right)}} \\
Q_{1}=\frac{V_{a} c_{\beta}\left[g_{1}-\frac{1}{m}\left(L+F_{t h r} s_{\alpha}\right)\right]+V_{w_{x}}\left(\frac{F_{z_{a}}}{m}+g c_{\theta} c_{\phi}\right)-V_{w_{z}}\left(\frac{F_{x_{a}}}{m}+\frac{F_{t h r}}{m}-g s_{\theta}\right)}{\left(V_{a} c_{\beta}\right)^{2}+2 V_{a} c_{\beta}\left(V_{w_{x}} c_{\alpha}+V_{w_{z}} s_{\alpha}\right)+V_{w_{x}}^{2}+V_{w_{z}}^{2}} \\
Q_{2}=\frac{V_{a}^{2} c_{\beta}\left[\frac{1}{m}\left(Y-F_{t h r} c_{\alpha} s_{\beta}\right)+g_{2}\right]+\left(\frac{F_{y_{a}}}{m}+g c_{\theta} s_{\phi}\right)\left[2 V_{a} c_{\beta}\left(V_{w_{x}} c_{\alpha}+V_{w_{z}} s_{\alpha}\right)+V_{w_{x}}^{2}+V_{w_{z}}^{2}\right]}{\left\{V_{a}^{2}+2 V_{a}\left[V_{w_{y}} s_{\beta}+c_{\beta}\left(V_{w_{x}} c_{\alpha}+V_{w_{z}} s_{\alpha}\right)\right]+V_{w_{x}}^{2}+V_{w_{y}}^{2}+V_{w_{z}}^{2}\right\} \sqrt{V_{a}^{2} c_{\beta}^{2}-V_{w_{y}}\left(2 V_{a} s_{\beta}+V_{w_{y}}\right)}} \\
\frac{-\left(\frac{F_{x_{a}}}{m}+\frac{F_{t h r}}{m}-g s_{\theta}\right)\left[V_{a}\left(V_{w_{y}} c_{\alpha} c_{\beta}+V_{w_{x}} s_{\beta}\right)+V_{w_{x}} V_{w_{y}}\right]-\left(\frac{F_{z_{a}}}{m}+g c_{\theta} c_{\phi}\right)\left[V_{a}\left(V_{w_{y}} s_{\alpha} c_{\beta}+V_{w_{z}} s_{\beta}\right)+V_{w_{y}} V_{w_{z}}\right]}{1} Q_{3}=\frac{1}{m}\left(F_{t h r} c_{\alpha} c_{\beta}-D\right)+\frac{1}{m V_{a}}\left[V_{w_{z}}\left(F_{z_{a}}+m g c_{\theta} c_{\phi}\right)+V_{w_{x}}\left(F_{x_{a}}+F_{t h r}-m g s_{\theta}\right)+V_{w_{y}}\left(F_{y_{a}}+m g c_{\theta} s_{\phi}\right)\right]
\end{gathered}
$$

which can be written as:

$$
\dot{R}=H(R) \Omega+Q(R)
$$

It can be easily verified that if the wind components are zero, the equations (11), (12) and (13) take the form:

$$
\begin{aligned}
\dot{\alpha} & =q-\tan (\beta)(p \cos (\alpha)+r \sin (\alpha))+\frac{1}{V_{a} \cos (\beta)}\left[g_{1}-\frac{1}{m}\left(L+F_{t h r} \sin (\alpha)\right)\right] \\
\dot{\beta} & =p \sin (\alpha)-r \cos (\alpha)+\frac{1}{V_{a}}\left[\frac{1}{m}\left(Y-F_{t h r} \cos (\alpha) \sin (\beta)\right)+g_{2}\right] \\
\dot{V}_{a} & =g_{3}+\frac{1}{m}\left(F_{t h r} \cos (\alpha) \cos (\beta)-D\right)
\end{aligned}
$$

The rotation speed components are related with the attitude angle rates by the Euler equations, given by:

$$
\left[\begin{array}{c}
\dot{\phi} \\
\dot{\theta} \\
\dot{\psi}
\end{array}\right]=\left[\begin{array}{ccc}
1 & t g_{\theta} s_{\phi} & t g_{\theta} c_{\phi} \\
0 & c_{\phi} & -s_{\phi} \\
0 & \frac{s_{\phi}}{c_{\theta}} & \frac{c_{\phi}}{c_{\theta}}
\end{array}\right]\left[\begin{array}{l}
p \\
q \\
r
\end{array}\right]
$$




\section{Guidance Dynamics}

Considering that the thrust force has only components in $x_{B}$, and knowing the relations for the aerodynamic forces in the body frame:

$$
\left[\begin{array}{c}
F_{x_{a}} \\
F_{y_{a}} \\
F_{z_{a}}
\end{array}\right]=L_{B W}\left[\begin{array}{c}
-D \\
Y \\
-L
\end{array}\right]
$$

and

$$
\left[\begin{array}{c}
D \\
Y \\
L
\end{array}\right]=\frac{1}{2} \rho S V_{a}^{2}\left[\begin{array}{l}
C_{D} \\
C_{Y} \\
C_{L}
\end{array}\right]
$$

The translational equations in the Earth frame are obtained by the 2nd Newton's law:

$$
\left[\begin{array}{c}
\ddot{x}_{E} \\
\ddot{y}_{E} \\
\ddot{z}_{E}
\end{array}\right]=L_{E B}\left[\begin{array}{c}
F_{x_{a}}+F_{t h r} \\
F_{y_{a}} \\
F_{z_{a}}
\end{array}\right] \frac{1}{m}+\left[\begin{array}{l}
0 \\
0 \\
g
\end{array}\right]
$$

\section{Actuator Dynamics}

Let a first-order model of the actuators be considered, assuming $\delta_{i}^{d}(i=$ ail, ele, rud $)$ as the desired positions of the control surfaces, and $\delta_{i}$ as the current positions of the control surfaces.

$$
\dot{\delta}_{i}=\frac{1}{\xi_{i}}\left(\delta_{i}^{d}-\delta_{i}\right)
$$

where $\xi_{i}$ are the time-constants. Also, the resultant thrust produced by the engines is supposed to behave as a first-order system, denoted by

$$
\dot{F}_{t h r}=\frac{1}{\xi_{T}}\left(F_{t h r}^{d}-F_{t h r}\right)
$$

where the $F_{t h r}^{d}$ is the desired thrust and $F_{t h r}$ the current thrust. Besides, the time-constants of the actuators keep the relation: $\xi_{T}>>\xi_{i}$.

\section{CONTROL}

\section{A. Attitude Control}

Taking the angular velocities as intermediary control variables, the necessary position of the actuators (deflection of aileron, elevator and rudder) as functions of the desired angular velocities must be determined, so the jerk vector of the angular velocities is obtained by differentiating one more time equation (10), leading to

$$
\left[\begin{array}{c}
\ddot{p} \\
\ddot{q} \\
\ddot{r}
\end{array}\right]=\frac{1}{2} \rho S I^{-1}\left\{V_{a}^{2}\left(\left[\begin{array}{c}
b \dot{C}_{l} \\
\bar{c} \dot{C}_{m} \\
b \dot{C}_{n}
\end{array}\right]+C_{\delta} \xi\left[\begin{array}{c}
\delta_{\text {ail }}^{d}-\delta_{\text {ail }} \\
\delta_{\text {ele }}^{d}-\delta_{\text {ele }} \\
\delta_{\text {rud }}^{d}-\delta_{\text {rud }}
\end{array}\right]\right)+2 V_{a} \dot{V}_{a}\left(\left[\begin{array}{c}
b C_{l} \\
\bar{c} C_{m} \\
b C_{n}
\end{array}\right]+C_{\delta}\left[\begin{array}{c}
\delta_{\text {ail }} \\
\delta_{\text {ele }} \\
\delta_{\text {rud }}
\end{array}\right]\right)\right\}-I^{-1} I_{n}\left[\begin{array}{c}
\dot{p} \\
\dot{q} \\
\dot{r}
\end{array}\right]
$$

with

$$
I_{n}=\left[\begin{array}{ccc}
-E q & (C-B) r-E p & (C-B) q \\
(A-C) r+2 E p & 0 & (A-C) p-2 E r \\
(B-A) q & (B-A) p+E r & E q
\end{array}\right]
$$

and

$$
\xi=\left[\begin{array}{ccc}
\frac{1}{\xi_{\text {ail }}} & 0 & 0 \\
0 & \frac{1}{\xi_{\text {ele }}} & 0 \\
0 & 0 & \frac{1}{\xi_{\text {rud }}}
\end{array}\right]
$$


where the aerodynamic moment coefficients dynamics are taken into account due to their close relation with $R$. On the other hand, control surfaces moment coefficients dynamics are neglected. Hence, differentiating (9), equation (23) can be rewritten as:

$$
\begin{aligned}
{\left[\begin{array}{c}
\ddot{p} \\
\ddot{q} \\
\ddot{r}
\end{array}\right]=} & \frac{1}{2} \rho S I^{-1}\left\{V_{a}^{2} C_{\delta} \xi\left[\begin{array}{c}
\delta_{\text {ail }}^{d}-\delta_{\text {ail }} \\
\delta_{\text {ele }}^{d}-\delta_{\text {ele }} \\
\delta_{\text {rud }}^{d}-\delta_{\text {rud }}
\end{array}\right]+V_{a}^{2} C_{c}\left[\begin{array}{c}
\dot{\alpha} \\
\dot{\beta} \\
\dot{V}_{a}
\end{array}\right]+2 V_{a} \dot{V}_{a}\left(\left[\begin{array}{c}
b C_{l} \\
\bar{c} C_{m} \\
b C_{n}
\end{array}\right]+C_{\delta}\left[\begin{array}{c}
\delta_{\text {ail }} \\
\delta_{\text {ele }} \\
\delta_{\text {rud }}
\end{array}\right]\right)\right\} \\
& +I^{-1}\left(\frac{1}{4} \rho S V_{a} C_{k}-I n\right)\left[\begin{array}{c}
\dot{p} \\
\dot{q} \\
\dot{r}
\end{array}\right]
\end{aligned}
$$

where

$$
\begin{gathered}
C_{c}=\left[\begin{array}{ccc}
0 & b C_{l_{\beta}} & -\frac{b^{2}}{2 V_{a}^{2}}\left(C_{l_{p}} p+C_{l_{r}} r\right) \\
\bar{c} C_{m_{\alpha}} & 0 & -\frac{\bar{c}^{2}}{2 V_{a}^{2}} C_{m_{q}} q \\
0 & b C_{n_{\beta}} & -\frac{b^{2}}{2 V_{a}^{2}}\left(C_{n_{p}} p+C_{n_{r}} r\right)
\end{array}\right] \\
C_{k}=\left[\begin{array}{ccc}
b^{2} C_{l_{p}} & 0 & b^{2} C_{l_{r}} \\
0 & \bar{c}^{2} C_{m_{q}} & 0 \\
b^{2} C_{n_{p}} & 0 & b^{2} C_{n_{r}}
\end{array}\right]
\end{gathered}
$$

The vector $\dot{R}$ can be taken from (15), and the vector $\dot{\Omega}$ from (10). Therefore, inverting the dynamics leads to an attitude control input denoted by:

$$
\begin{aligned}
{\left[\begin{array}{c}
\delta_{\text {ail }}^{d} \\
\delta_{\text {ele }}^{d} \\
\delta_{\text {rud }}^{d}
\end{array}\right]=} & \frac{1}{V_{a}^{2}} \xi^{-1} C_{\delta}^{-1}\left\{\frac{2 I}{\rho S}\left[\begin{array}{c}
\tau_{p} \\
\tau_{q} \\
\tau_{r}
\end{array}\right]-\frac{2}{\rho S}\left(\frac{1}{4} \rho S V_{a} C_{k}-I n\right)\left[\begin{array}{c}
\dot{p} \\
\dot{q} \\
\dot{r}
\end{array}\right]-2 V_{a} \dot{V}_{a}\left(\left[\begin{array}{c}
b C_{l} \\
\bar{c} C_{m} \\
b C_{n}
\end{array}\right]+C_{\delta}\left[\begin{array}{c}
\delta_{\text {ail }} \\
\delta_{\text {ele }} \\
\delta_{\text {rud }}
\end{array}\right]\right)\right. \\
& \left.-V_{a}^{2} C_{c}\left[\begin{array}{c}
\dot{\alpha} \\
\dot{\beta} \\
\dot{V_{a}}
\end{array}\right]\right\}+\left[\begin{array}{c}
\delta_{\text {ail }} \\
\delta_{\text {ele }} \\
\delta_{\text {rud }}
\end{array}\right]
\end{aligned}
$$

where the wind effects appear in the terms involving $\dot{\alpha}, \dot{\beta}$ and $\dot{V}_{a}$. Also, desired dynamics for the angular velocities are proposed:

$$
\left[\begin{array}{c}
\tau_{p} \\
\tau_{q} \\
\tau_{r}
\end{array}\right]=\left[\begin{array}{c}
-k_{1}\left(p-p^{d}\right)-k_{2}\left(\dot{p}-\dot{p}^{d}\right)+\ddot{p}^{d} \\
-k_{3}\left(q-q^{d}\right)-k_{4}\left(\dot{q}-\dot{q}^{d}\right)+\ddot{q}^{d} \\
-k_{5}\left(r-r^{d}\right)-k_{6}\left(\dot{r}-\dot{r}^{d}\right)+\ddot{r}^{d}
\end{array}\right]
$$

for $k_{i}>0(i=1, \ldots 6)$ as gains, chosen in order to assure asymptotical convergence of the variables to their desired values. The feasibility of this approach depends on the singularity of $C_{\delta}$, the matrix involving the aerodynamic coefficients (assumed to be known thanks to experimental data, airflow simulations, or any other method) due to the control surfaces, aspect that can be handled.

\section{B. Position Control}

In order to assure trajectory tracking, angular velocities and thrust required to follow a reference trajectory need to be obtained, so after differentiating equation (20), the jerk vector of the position in $F_{E}$ is obtained.

$$
\left[\begin{array}{c}
x_{E}^{(3)} \\
y_{E}^{(3)} \\
z_{E}^{(3)}
\end{array}\right]=\frac{\dot{L}_{E B}}{m}\left[\begin{array}{c}
F_{x_{a}}+F_{t h r} \\
F_{y_{a}} \\
F_{z_{a}}
\end{array}\right]+\frac{L_{E B}}{m}\left[\begin{array}{c}
\dot{F}_{x_{a}}+\dot{F}_{t h r} \\
\dot{F}_{y_{a}} \\
\dot{F}_{z_{a}}
\end{array}\right]+\frac{-\dot{m}}{m^{2}} L_{E B}\left[\begin{array}{c}
F_{x_{a}}+F_{t h r} \\
F_{y_{a}} \\
F_{z_{a}}
\end{array}\right]
$$

Considering that the mass rate of change is very small compared to the aircraft total mass, the term containing $\frac{-\dot{m}}{m^{2}}$ is neglected. Also, writing the Euler property as

$$
\begin{aligned}
\dot{L}_{E B} & =L_{E B}\left[\begin{array}{ccc}
0 & -r & q \\
r & 0 & -p \\
-q & p & 0
\end{array}\right] \\
& =M_{p} p+M_{q} q+M_{r} r
\end{aligned}
$$


where

$$
\begin{gathered}
M_{p}=\left[\begin{array}{ccc}
0 & c_{\phi} s_{\theta} c_{\psi}+s_{\phi} s_{\psi} & c_{\phi} s_{\psi}-s_{\phi} s_{\theta} c_{\psi} \\
0 & c_{\phi} s_{\theta} s_{\psi}-s_{\phi} c_{\psi} & -s_{\phi} s_{\theta} s_{\psi}-c_{\phi} c_{\psi} \\
0 & c_{\phi} c_{\theta} & -s_{\phi} c_{\theta}
\end{array}\right] \\
M_{q}=\left[\begin{array}{ccc}
-c_{\phi} s_{\theta} c_{\psi}-s_{\phi} s_{\psi} & 0 & c_{\theta} c_{\psi} \\
-c_{\phi} s_{\theta} s_{\psi}+s_{\phi} c_{\psi} & 0 & c_{\theta} s_{\psi} \\
-c_{\phi} c_{\theta} & 0 & -s_{\theta}
\end{array}\right] \\
M_{r}=\left[\begin{array}{ccc}
s_{\phi} s_{\theta} c_{\psi}-c_{\phi} s_{\psi} & -c_{\theta} c_{\psi} & 0 \\
s_{\phi} s_{\theta} s_{\psi}+c_{\phi} c_{\psi} & -c_{\theta} s_{\psi} & 0 \\
s_{\phi} c_{\theta} & s_{\theta} & 0
\end{array}\right]
\end{gathered}
$$

and defining the vector $F_{a b}=\left[F_{x_{a}}+F_{t h r}, F_{y_{a}}, F_{z_{a}}\right]^{T}$, equation (27) can be rewritten as:

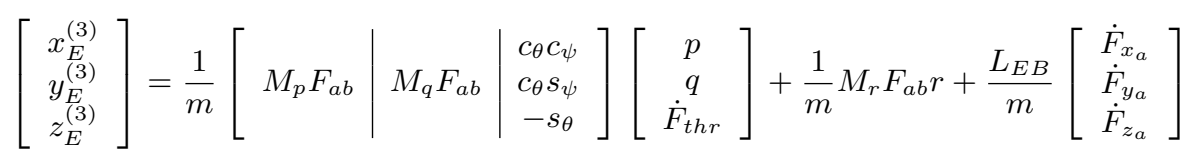

where

$$
\left[\begin{array}{c}
\dot{F}_{x_{a}} \\
\dot{F}_{y_{a}} \\
\dot{F}_{z_{a}}
\end{array}\right]=\left[\begin{array}{ccc}
\frac{\partial F_{x_{a}}}{\partial \alpha} & \frac{\partial F_{x_{a}}}{\partial \beta} & \frac{\partial F_{x_{a}}}{\partial V_{a}} \\
0 & \frac{\partial F_{y_{a}}}{\partial \beta} & \frac{\partial F_{y_{a}}}{\partial V_{a}} \\
\frac{\partial F_{z_{a}}}{\partial \alpha} & \frac{\partial F_{z_{a}}}{\partial \beta} & \frac{\partial F_{z_{a}}}{\partial V_{a}}
\end{array}\right] \dot{R}+\left[\begin{array}{c}
\frac{\partial F_{x_{a}}}{\partial \rho} \\
\frac{\partial F_{y_{a}}}{\partial F_{2}} \\
\frac{\partial F_{z_{a}}}{\partial \rho}
\end{array}\right] \dot{\rho}
$$

where $\dot{R}$ is obtained from equation (15). Thus, taking as guidance inputs the vector $\left[p, q, \dot{F_{t h r}}\right]^{T}$, the inversion of the dynamics can be proposed in the form:

$$
\left[\begin{array}{c}
p \\
q \\
F_{t h r}
\end{array}\right]=m\left[\begin{array}{l|l|l}
M_{p} F_{a b} & M_{q} F_{a b} & \begin{array}{c}
c_{\theta} c_{\psi} \\
c_{\theta} s_{\psi} \\
-s_{\theta}
\end{array}
\end{array}\right]^{-1}\left(\left[\begin{array}{c}
\tau_{x} \\
\tau_{y} \\
\tau_{z}
\end{array}\right]-\frac{1}{m} M_{r} F_{a b} r-\frac{L_{E B}}{m}\left[\begin{array}{c}
\dot{F}_{x_{a}} \\
\dot{F}_{y_{a}} \\
\dot{F}_{z_{a}}
\end{array}\right]\right)
$$

where the wind effects are involved in the vector $\left[\dot{F}_{x_{a}}, \dot{F}_{y_{a}}, \dot{F}_{z_{a}}\right]^{T}$, and desired behaviour of the position is proposed as:

$$
\left[\begin{array}{l}
\tau_{x} \\
\tau_{y} \\
\tau_{z}
\end{array}\right]=\left[\begin{array}{l}
-d_{1}\left(x-x^{d}\right)-d_{2}\left(\dot{x}-\dot{x}^{d}\right)-d_{3}\left(\ddot{x}-\ddot{x}^{d}\right)+\dddot{x}^{d} \\
-d_{4}\left(y-y^{d}\right)-d_{5}\left(\dot{y}-\dot{y}^{d}\right)-d_{6}\left(\ddot{y}-\ddot{y}^{d}\right)+\dddot{y}^{d} \\
-d_{7}\left(z-z^{d}\right)-d_{8}\left(\dot{z}-\dot{z}^{d}\right)-d_{9}\left(\ddot{z}-\ddot{z}^{d}\right)+\dddot{z}^{d}
\end{array}\right]
$$

for $d_{i}>0(i=1, \ldots 9)$ as gains chosen in order to assure asymptotical convergence of the variables to their desired values. The feasibility of this approach requires the invertibility of the matrix:

$$
M_{p q T}=\left[\begin{array}{l|l|l}
M_{p} F_{a b} & M_{q} F_{a b} & \begin{array}{c}
c_{\theta} c_{\psi} \\
c_{\theta} s_{\psi} \\
-s_{\theta}
\end{array}
\end{array}\right]
$$

which in the case in where $\phi \approx \psi \approx 0$, the determinant of this matrix is given by:

$$
\left|M_{p q T}\right|=s_{\theta} F_{y_{a}}+\left(s_{\theta}\left(F_{x_{a}}+F_{t h r}\right)-c_{\theta} F_{z_{a}}\right)\left(s_{\theta} F_{z_{a}}\right)+c_{\theta}\left(c_{\theta}\left(F_{x_{a}}+F_{t h r}\right)+s_{\theta} F_{z_{a}}\right) F_{z_{a}}
$$

and, when considered $\theta \approx 0$, a singularity appears for $F_{x_{a}}+F_{t h r}=0$. This is the case when the plane is cruising at constant speed. Therefore, in order to manage this singularity, the controller can be switched for another approach, or as considered in this work, the desired angular velocities are switched to zero. 


\section{Simulation Results}

A six degree of freedom model for a wide body transportation aircraft has been developed in Matlab to provide simulation results with the adopted control law as well as the sensitivity analysis, the aircraft parameters were chosen close enough to emulate a Boeing 737-200 (described in the Nomenclature), flying in an International Standard Atmosphere model with uniform gravity. The time response of actuators used to move the control surfaces is $50 \mathrm{~ms}$. To obtain the values of the aerodynamic coefficients, a two-layer feed-forward neural networks with sigmoid hidden neurons and linear output neurons were trained using data bases obtained from the United States Air Force (USAF) Stability and Control Digital DATCOM (Data Compendium) [16], which is a Public Domain Aeronautical Software that compute the static stability, control and dynamic derivative characteristics of fixed-wing aircrafts using the methods contained in the USAF Stability and Control DATCOM, furthermore, the data sets were compared and refined using the JSBSim open source Flight Dynamics Models (FDM) [17], used in several open source simulators and also employed to drive the motion-base research simulators of many universities worldwide. The training, validation and test sets were 70, 15 and 15 percent respectively of the available data for each aerodynamic coefficient. The training algorithm used was Bayesian Regularization and the number of hidden neurons was selected by trial and error trying to improve the performance as much as possible. The initial mass of the airplane is $50,000 \mathrm{~kg}$ and it is supposed to follow a dynamic as:

$$
\dot{m}=-\mu F_{t h r}
$$

Where the thrust specific fuel consumption involves the coefficients $c_{f 1}=2.854 \times 10^{-5} \mathrm{~kg} /(N \cdot \mathrm{m})$ and $c_{f 2}=8,693.36 \mathrm{~m} / \mathrm{s}$, as well as the airspeed[18], expressed by:

$$
\mu=c_{f 1}\left(1-\frac{V_{a}}{c_{f 2}}\right) \frac{V_{a}}{1,943.84}
$$

When the control surfaces are jammed at zero degrees, and enough constant thrust to hold a longitudinal flight is set, the behaviour of the aircraft is a stable, with decreasing phugoid mode with a $100 \mathrm{~s}$ period. Regarding to the attitude, in figure 1(a) are shown the behaviours of the angular velocities when the
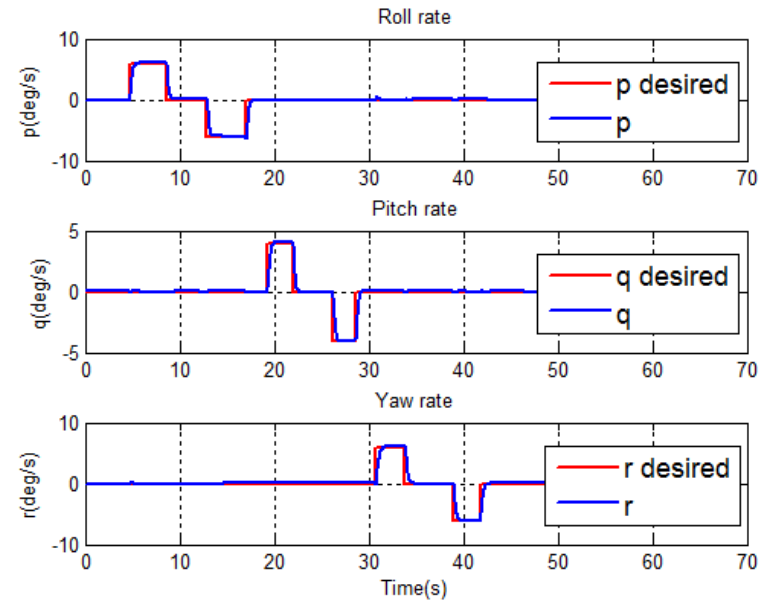

(a) Attitude response to commanded angular rates
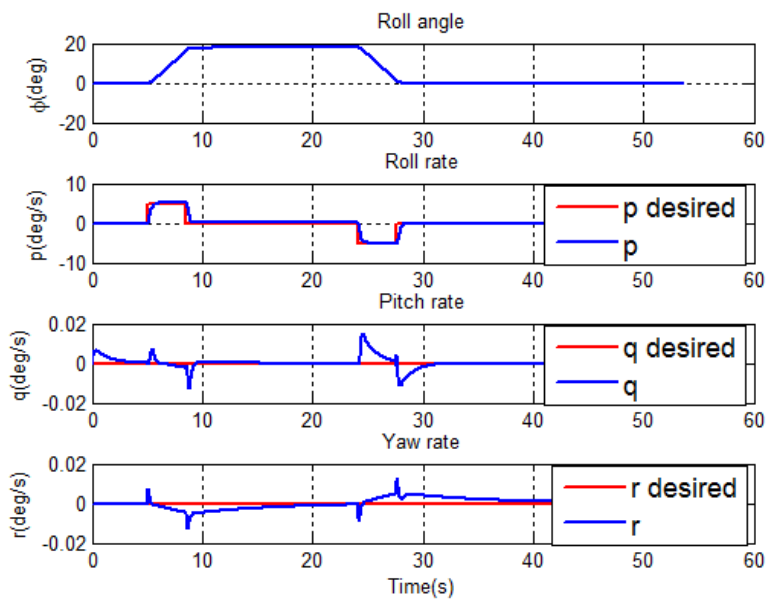

(b) Lateral displacement using roll

Fig. 1. Attitude control test.

attitude controller commands certain movements. Angular rates of $\pm 6 \mathrm{deg} / \mathrm{s}$ were commanded to roll and yaw, while a $\pm 4 \mathrm{deg} / \mathrm{s}$ rate for pitch. The influence that a yawing motion has on the roll rate and 

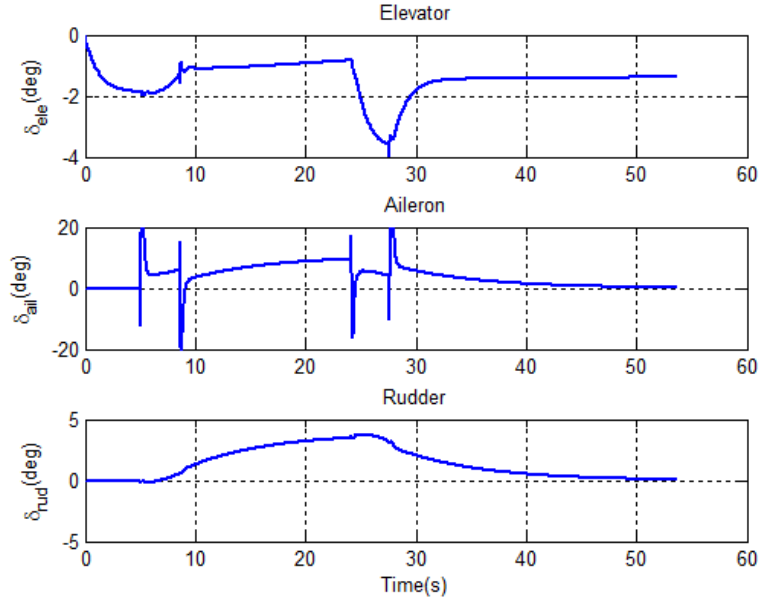

(a) Deflection of control surfaces for lateral displacement.
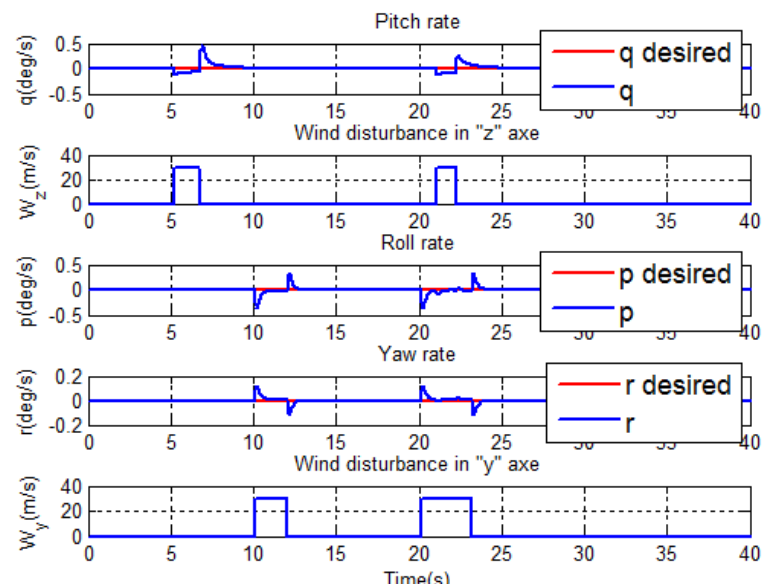

(b) Attitude response to disturbances.

Fig. 2. Control effort and rejection of perturbations.

viceversa can be nullified or controlled, which plays a key roll when coordinated turns are performed. In figure 1(b), a lateral displacement using roll is simulated, holding a fixed altitude and a constant yaw angle. In order to achieve this task, the deflection of the control surfaces of the aircraft is depicted in figure 2(a). Moreover, the behaviour of the attitude under some disturbances is examined by applying wind gusts of $30 \mathrm{~m} / \mathrm{s}\left(58.3\right.$ knots) (see figure 2(b)). The first disturbance is applied around $5 \mathrm{~s}$ in $z_{B}$ direction, which produces an asymmetric disturbance in pitch. Then, a second disturbance is applied around second 10 in $-y_{B}$ direction, which affects roll and yaw rates, as expected. Finally, a disturbance with components in both mentioned directions is applied around the second 21. Note that for the moment, the error produced in position by these wind gusts is not examined and only a zero angular rate is desired to be kept under these disturbances. It is worth to mention that during flight, constant large variations of the Thrust are not desired, so in this work, Thrust is considered constant, resulting in a longitudinal speed of $250 \mathrm{~m} / \mathrm{s}$.

In order to test de guidance controller, figure 3(a) shows the trajectory of the aircraft when different desired positions are commanded. An increase in altitude of $100 \mathrm{~m}$ and return to its original altitude, as well as a lateral displacement of $100 \mathrm{~m}$ and return to the original position are demanded. Next, a decrease of $10 \mathrm{~m}$ in altitude and a lateral displacement of $50 \mathrm{~m}$ in the opposite direction than the first one are commanded. Finally, the aircraft is asked to return to the original altitude and original $y$ position. The figure 3(b) displays the 3D trajectory of the described flight. The control approach also assures a climb and descent rate under 1,400 ft/min, as well as velocity in lateral displacements under $2,200 \mathrm{ft} / \mathrm{min}$. It is a key point to remark that as control inputs for guidance are the angular velocities, if an angular velocity is commanded for a relatively long period of time, this will derive in constant and maybe undesired turning of the aircraft. In order to avoid this problem, when Euler angles reach a certain magnitude, the angular velocity is forced to become zero until an angular velocity in the opposite direction is commanded. The saturation of the Euler angles are: $\phi\left\{-30^{\circ}, 30^{\circ}\right\} ; \theta\left\{-15^{\circ}, 25^{\circ}\right\}$; $\psi\{-\pi, \pi\}$.

Let the position errors produced by wind gusts be examined, taking into account that non-zero angular rates will allow the aircraft to reject perturbations and achieve the guidance objectives. In figure 4, wind gusts endowed with small turbulence (using a Dryden wind turbulence model) are applied, showing the effects on $y$ and $z$ position errors as well as the groundspeed, which has no control due to the constant Thrust. 

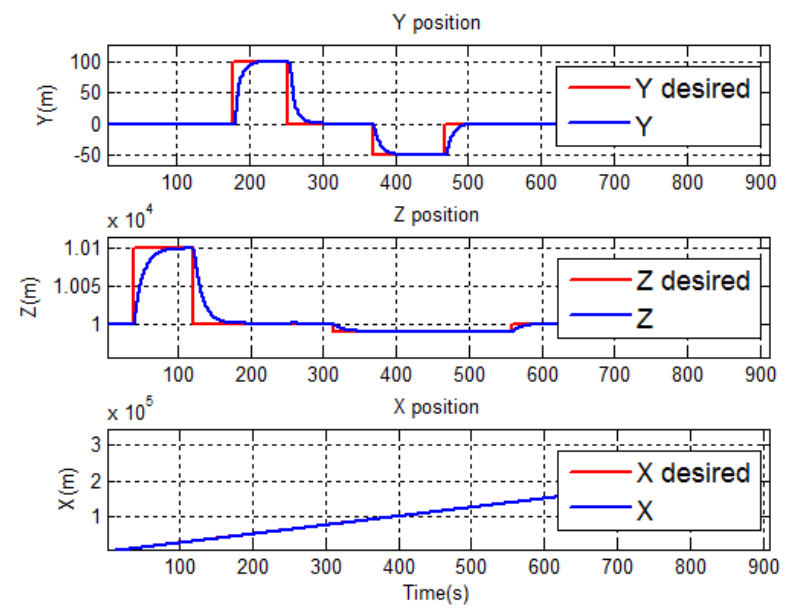

(a) Position response to commanded positions.

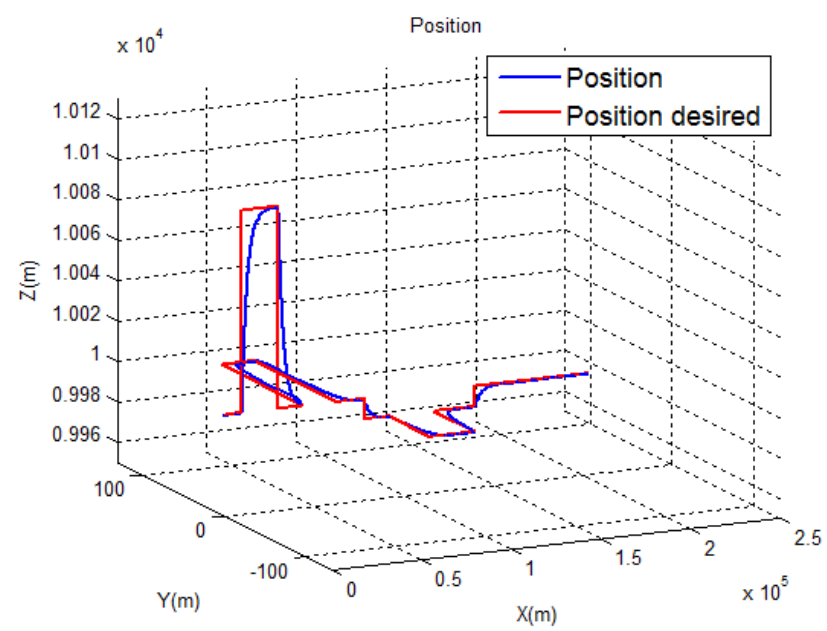

(b) 3D Trajectory of guidance control test.

Fig. 3. Guidance control test
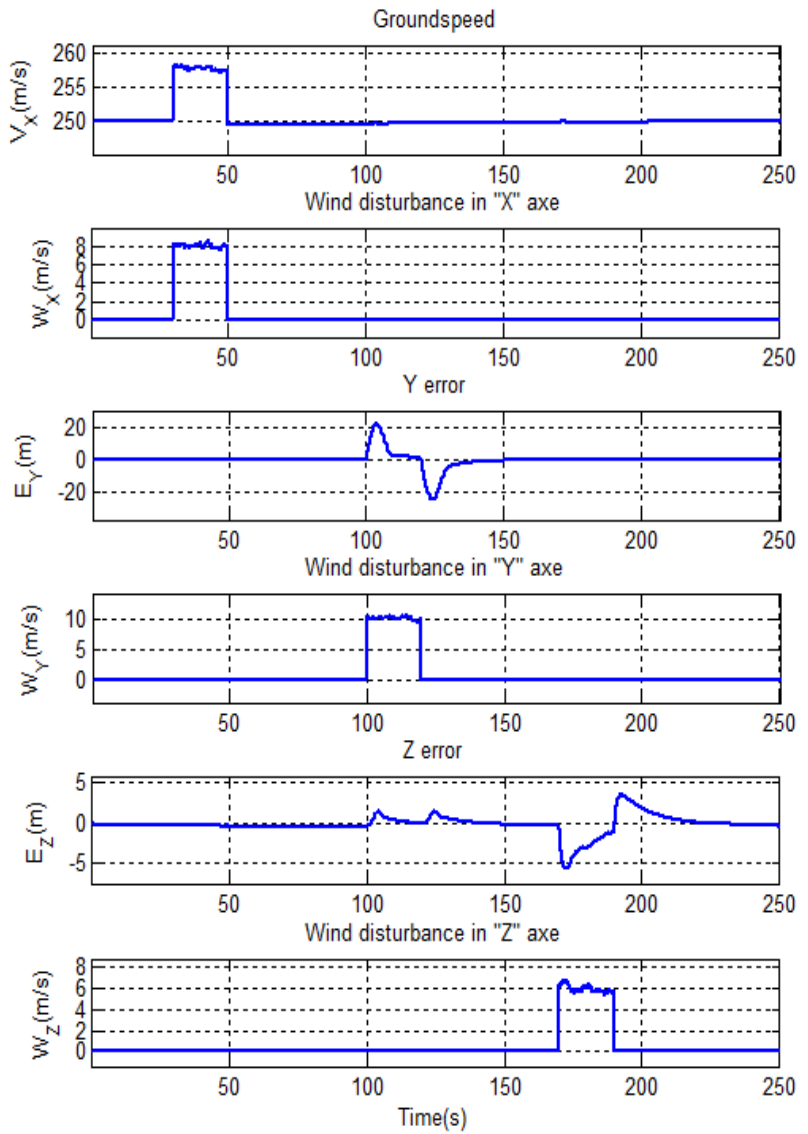

Fig. 4. Position response to disturbances. 


\section{Towards Sensitivity Analysis Through Simulation}

So far, the controller is assumed to have full knowledge of wind disturbances (by estimations, measures or forecasts available), but in reality, the use of corrupted measurements, approximated mathematical models, or uncertainty in parameters leave room for errors in the control, which can be studied through a sensitivity analysis. For our study, it is assumed that wind knowledge by the
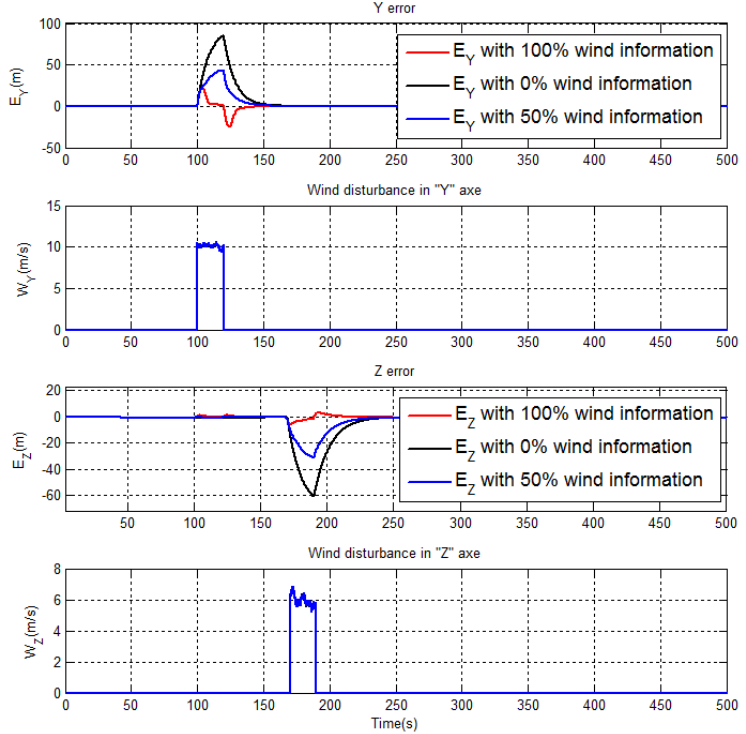

(a)
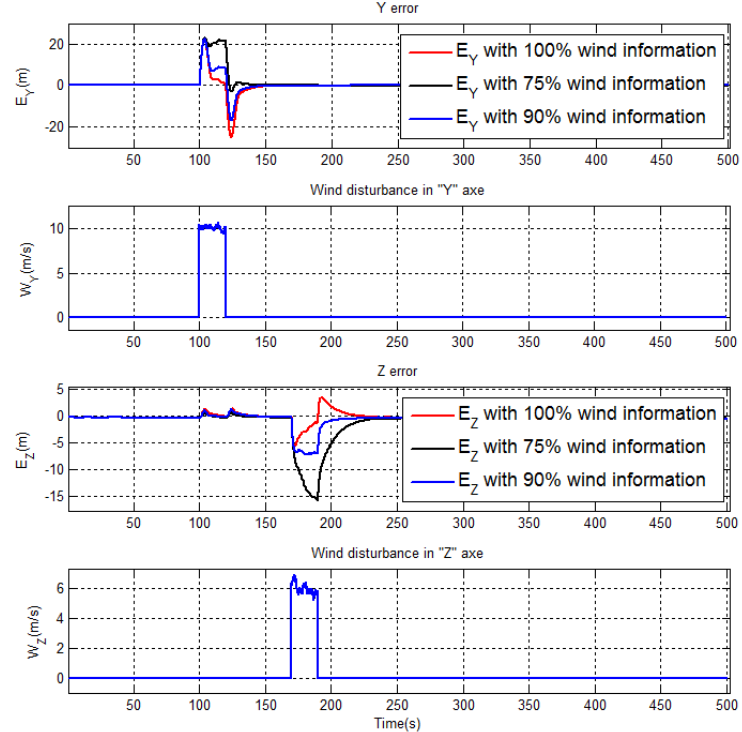

(b)

Fig. 5. Wind knowledge effect on position errors.

TABLE I

ERROR DEPENDING ON WIND KNOWLEDGE

\begin{tabular}{ccccc}
\hline \hline & \multicolumn{2}{c}{$\mathbf{y}$} & \multicolumn{2}{c}{$\mathbf{z}$} \\
\cline { 2 - 5 } Wind Knowledge (\%) & MSE & MAE & MSE & MAE \\
\hline 100 & 10.2760 & 0.6731 & 0.7806 & 0.5767 \\
90 & 8.4628 & 0.6787 & 2.0858 & 0.6993 \\
80 & 13.8191 & 0.8001 & 5.8818 & 1.0335 \\
70 & 23.0642 & 1.0926 & 11.7237 & 1.2839 \\
60 & 35.9015 & 1.4416 & 20.3080 & 1.6094 \\
50 & 55.9952 & 1.8394 & 31.2249 & 1.9199 \\
40 & 78.7370 & 2.2092 & 44.1320 & 2.2091 \\
30 & 107.6038 & 2.6044 & 59.4018 & 2.4883 \\
20 & 140.3636 & 2.9848 & 77.5264 & 2.7895 \\
10 & 177.5873 & 3.3675 & 97.0740 & 3.0764 \\
0 & 220.3000 & 3.7540 & 118.4172 & 3.3786 \\
\hline \hline
\end{tabular}

controller is the main contributor to position error improvements. Note that by wind knowledge, we refer to the accuracy of wind gust magnitude and not the direction. So, a $70 \%$ of wind knowledge when a wind gust of $10 \mathrm{~m} / \mathrm{s}$ is considered, will be $7 \mathrm{~m} / \mathrm{s}$ in the same direction. So that, the transport aircraft of Section IV flying at 10,000 m with a constant groundspeed of $250 \mathrm{~m} / \mathrm{s}$ under some wind disturbances is used, and endowing the controller with different wind knowledge, the position errors are examined. The position errors for $y$ and $z$ with $0 \%, 50 \%, 75 \%, 90 \%$ and $100 \%$ of wind knowledge are displayed in 
figure 5, where the error magnitude decreases when wind knowledge increases. This means that as the components of equation (15) used for the control approach tend to equations (16a), (16b) and (16c), the controller will decrease its performance drastically, corroborating that the reactiveness of the control law (and hence, the position errors), is sensitive to wind knowledge. In Table I, the Mean Squared Error (MSE) and the Mean Absolute Error (MAE) in $y$ and $z$ axes were computed in order to provide a clear idea of the wind sensitivity w.r.t. aircraft position. The disturbances, simulation time $(500 \mathrm{~s})$ and sample time $(1 / 30 \mathrm{~s})$ are the same as the ones to obtain figure 5 . It is worth to note that even if full wind knowledge is achieved, the control effort will always remain bounded within the typical constraints for transport aircraft.

\section{CONCLUSION}

Depending on each application or requirement, a maximal wind uncertainty can be allowed in order to do not exceed a desired error in position, proposed by the user. This wind uncertainty can be translated into wind sensors accuracy, wind estimations accuracy or up-to-date forecasted wind. The control law used in this work can be easily replaced by other control law to compare their sensitivity to wind. A safety envelope for a single aircraft or a group of aircrafts can be redefined, as errors will depend on wind knowledge accuracy, allowing to create compact aircraft clusters and decrease the workload for air traffic controllers. In this paper, a framework for wind sensitivity analysis is described in order to test different control laws as well as different wind uncertainties and their effects in guidance errors, quantifying its performance by computing the mean squared error and mean absolute error. A mathematical model and a NDI approach considering wind disturbances in AoA and sideslip angle, as well as in airspeed, was developed and tested in a six degrees of freedom Matlab simulation. 


\section{REFERENCES}

[1] FAA. Next generation air transportation system. Https://www.faa.gov/nextgen/.

[2] EUROCONTROL. Single european sky atm research joint undertaking. Http://www.sesarju.eu/.

[3] J. J. E. Slotin and W. Li, Applied Nonlinear Control. Englewood Cliffs, NJ, Ch 6: Prentice Hall, 1991.

[4] H. K. Khalil, Nonlinear Systems, 3rd ed. Upper Saddle River, NJ, Ch 13: Prentice Hall, 2002.

[5] S. A. Snell, D. F. Enns, and W. L. Garrard, "Nonlinear inversion flight control for a supermaneuverable aircraft," AIAA Journal of Guidance, Control, and Dynamics, vol. 15, no. 4, pp. 976-984, July 1992.

[6] T. Lombaerts, G. Looye, Q. Chu, and J. Mulder, "Pseudo control hedging and its application for safe flight envelope protection," AIAA Guidance, Navigation, and Control Conference, August 2010.

[7] R. Rysdyk and A. J. Calise, "Robust nonlinear adaptive flight control for consistent handling qualities," IEEE Transactions on Control Systems Technology, vol. 13, no. 6, pp. 896-910, November 2005.

[8] I. Hameduddin and A. H. Bajodah, "Generalized dynamic inversion control for aircraft constrained trajectory tracking applications," American Control Conference (ACC), pp. 4599-4606, June 2012.

[9] S. Schnelle and J. Wang, "Sensitivity analysis of human driving characteristics on road and driving conditions for active vehicle control systems," IEEE International Conference on Systems, Man, and Cybernetics, pp. 2482-2487, October 2014.

[10] A. Janon, M. Nodet, C. Prieur, and C. Prieur, "Global sensitivity analysis for the boundary control of an open channel," IEEE Conference on Decision and Control, pp. 589-594, December 2014.

[11] K. A. Evans, "Sensitivity analysis for control parameter determination for a nonlinear cable-mass system," 2009 American Control Conference (ACC), pp. 4091-4096, June 2009.

[12] M. Kanno, S. Hara, R. Nakamura, and M. Matsubara, "Algebraic approach to sensitivity analysis in optimal feedback control system design,” IEEE International Symposium on Computer-Aided Control System Design, pp. 1696-1701, September 2010.

[13] B. Etkin and L. D. Reid, Dynamics of Flight, Stability and Control, 3rd ed. John Wiley and Sons, 1996.

[14] B. L. Stevens and F. L. Lewis, Aircraft Control and Simulation, 2nd ed. John Wiley and Sons, 1992.

[15] T. Lombaerts, H. Huisman, Q. Chu, J. Mulder, and D. Joosten, "Nonlinear reconfiguring flight control based on on-line physical model identification," AIAA Journal of Guidance, Control, and Dynamics, vol. 32, no. 3, pp. 727-748, May-June 2009.

[16] Public Domain Aeronautical Software. Http://www.pdas.com/datcom.htm.

[17] Public Domain Aeronautical Software. Http://jsbsim.sourceforge.net/.

[18] User Manual for the Base of Aircraft Data (BADA), European Organisation for the Safety of Air Navigation, April 2010. 